\title{
Comparison between Supine and Lateral Positions In Relation To Ease of Mask Ventilation and Laryngoscopic Views With or Without Burp Maneuver in General Anaesthesia
}

\author{
Suranjith Sorake ${ }^{1}$, Harish Hegde ${ }^{2}$, Ayaskant Sahoo $^{3}$ \\ ${ }^{I}$ (Assistant professor, Yenepoya Medical College, Mangalore, Karnataka, India) \\ ${ }^{2}$ (Professor, Yenepoya Medical College, Mangalore, Karnataka, India) \\ ${ }^{3}$ (Post graduate, Yenepoya Medical College, Mangalore, Karnataka, India)
}

\begin{abstract}
In patients undergoing surgeries, airway is most often secured with the endotracheal tube under general anaesthesia in supine position. Then position of the patient is changed according to the surgical requirement. Many surgical procedures are done in lateral position under regional anaesthesia. Because of various reasons we may have to supplement with general anaesthesia using endotracheal tube. In many situations turning the patient to supine may be difficult because of the ongoing surgery and endotracheal intubation has to be done in the lateral position. LMA and ILMA are considered safe and first choice for securing the airway in lateral position but it requires more expertise and positive pressure ventilation may be difficult. Direct laryngoscopy is still the fastest and easiest method of securing airway as it is an instrument familiar to all anaesthesiologists and readily available at every anaesthetizing location. In this study we compare the efficacy of BURP (backward, upward, rightward pressure) maneuver in improving the visualization of vocal cords in both supine and lateral positions. We also compared the ease of mask ventilation in supine and lateral positions.
\end{abstract}

Keywords: BURP maneuver, lateral, supine, mask ventilation,

\section{Introduction:}

Principal causes of difficult laryngoscopy are anterior larynx, retrusion of tongue, protrusion of upper teeth. BURP maneuver shifts the larynx posteriorly and superiorly and improves the exposure by advancing the larynx around the base of the tongue and towards the oropharynx. With larynx in this position, the glottis may lie in a more vertical laryngoscopic line of vision that would be less affected by both the base of the tongue and the upper teeth. The moderate rightward displacement produced by the BURP maneuver may further enhance the view by placing the glottis in the more open visual pathway along the right side of the cavity which is created as the laryngoscope blade sweeps the tongue towards left. ${ }^{1,2,3}$ The ease of mask ventilation in supine and lateral positions was compared and graded as easy, difficult but adequate and inadequate. Easy: Adequate ventilation possible by one person and no increase in fresh gas flow (FGF) or use of oral airway required. Difficult but adequate: Adequate ventilation possible but needs two people and an in case in FGF or use of oral airway. Inadequate: If mask ventilation was inadequate in spite of the above measures in the supine position. Those patients were excluded from study.

\section{Material and Methods:}

We conducted a prospective, randomized and controlled study. We conducted the study on 72 patients posted for elective surgeries under general anaesthesia in our hospital. Ethical committee clearance was obtained prior to start of the study. Informed written consent was taken from all patients. All patients were kept nil by mouth for six hours for solids and three hours for clear fluids

Inclusion criteria: The patients included in the study were in age group of 18-60 years,either gender, ASA PS(American society of anaesthesiologists physical status)Class I or II, undergoing elective general anaesthesia and requiring endotracheal intubation . Exclusion criteria :Patients who were excluded were belonging to any following group. Known or predicted difficult airway, Mallampati class III or IV, Mouth opening $<2$ fingers breadths, Thyromental distance $<3$ fingers breadths, Temporomandibular joint $<1$ finger breadth, Buckteeth, edentulous patients, restricted neck movements, presence of beard, pregnancy, any spine disease or distorted spinal anatomy, any specific contraindications for turning patients to lateral position, obese patients (BMI $>30$ ). All patients were premedicated with Tab Diazepam ( $5 \mathrm{mg}$ if weight is $<50 \mathrm{~kg}$ and $10 \mathrm{mg}$ for weight $>50 \mathrm{~kg}$ ), Tab Ranitidine $150 \mathrm{mg}$ and Tab Metoclopramide $10 \mathrm{mg}$, on the night before and on the morning of surgery. On the day of surgery the patients were shifted to the operating room (OR), intravenous (IV) access secured and baseline monitoring commenced. Monitoring included (electrocardiogram Lead II and $\mathrm{V}_{5}$ ), pulse oximetry and noninvasive blood pressure. The patients were placed supine with a pillow under the occiput to 
achieve 'sniffing' position. All patients were pre-oxygenated with oxygen at a flow rate of $100 \mathrm{ml} \mathrm{kg}^{-1} \mathrm{~min}^{-1}$ for 3 minutes. Anaesthesia was induced with fentanyl $2 \mu \mathrm{g} \mathrm{kg}^{-1}$ IV followed by propofol $2 \mathrm{mg} \mathrm{kg}^{-1} \mathrm{IV}$. The ability to mask ventilate was checked and if found satisfactory, neuromuscular blockade was achieved with vecuronium $0.1 \mathrm{mg} \mathrm{kg}^{-1}$. All patients were ventilated by face mask in the supine and lateral position with halothane or isoflurane in oxygen for 3 minutes. The ease of mask ventilation was then assessed. Adequate neuromuscular blockade was ensured. A senior anaesthesiologist ( $>5$ years experience in anaesthesia) performed direct laryngoscopy to grade degree of laryngeal visualization as per modified Cormack and Lehane classification, initially without and then, with BURP maneuver. If the laryngoscopic grade was Grade III or IV even with BURP maneuver, the patients were excluded from the study. The patients were randomly assigned (using random number generator) to one of two groups: Group $S$ : Conventional Mask Ventilation and direct laryngoscopy in supine position. Group L: Conventional Mask Ventilation and direct laryngoscopy and endotracheal intubation in position. A bolus dose of propofol 10-20 mg was given to deepen the anaesthetic. The patients were turned into either supine or lateral position according to the group assigned. Pillows were kept under the head in such a way that the sagittal axis of head and neck were parallel to the table top. All patients were ventilated by face mask in the lateral position for $1 \mathrm{~min}$ and the ease recorded. Another bolus dose of propofol 10-20 mg was given (if needed) to ensure adequate depth of anaesthesia. The senior anaesthesiologist performed direct laryngoscopy in the lateral position initially without and then with BURP maneuver

\section{Results:}

The time to laryngoscopy was statistically significant difference in supine and lateral positions, where laryngoscopy needed about 6 seconds longer in lateral position. Though this was statistically significant, clinically it was insignificant. This was probably due acclimatization to a new technique and the fact that the blade slipped in a few cases.

When the laryngoscopic view was compared in supine without the application of BURP vs application of BURP, all patients had a laryngoscopic grading between $1(\mathrm{n}=14,38 \%) 2 \mathrm{a}(\mathrm{n}=16,44.4 \%), 2 \mathrm{~b}(\mathrm{n}=6,18 \%)$ in supine position without the application of BURP after which it improved to a grading between $1(\mathrm{n}=36,86 \%)$ and $2 \mathrm{a}(\mathrm{n}=6,14 \%)$ with the application of BURP. There was a statistically significant improvement $\left(\mathrm{X}^{2}=16.364\right.$ ;PVALUE 0.003 in the thelaryngoscopic grade with the application of BURP .

The laryngoscopic view was compared in lateral position without the application of BURP vs application of BURP(Backward, Upward, Rightward Pressure), all patients had a laryngoscopic grading between $1(\mathrm{n}=19,41.3 \%), 2 \mathrm{a}(\mathrm{n}=11,29.5 \%)$ and $2 \mathrm{~b}(\mathrm{n}=16,34.7 \%)$ in lateral position without the application of BURP after which it improved to a grading between $1(\mathrm{n}=30,86.1 \%)$ and $2 \mathrm{a}(\mathrm{n}=6,14 \%)$ with the application of BURP. There was a statistically significant improvement $\left(X^{2}=9.939\right.$; P VALUE 0.007$)$ in thelaryngoscopic grade with the application of BURP in the lateral postion.

We evaluated the effect of BURP maneuver on laryngoscopic grade. In the supine position, $82 \%$ of the patients had a Grade 1 of 2 a views which changed to $100 \%$ with BURP maneuver. In the lateral position, only $70 \%$ of patients had a Grade 1 or $2 \mathrm{a}$ views which changed to $100 \%$ with BURP maneuver. The improvement in laryngoscopic grade was found to be statistically very highly significant as also clinically significant with the application of the BURP maneuver.Thelaryngoscopy time between the supine $(6.2500 \pm 2.22 \mathrm{~s})$ and lateral $(13.3056 \pm 4.59079 \mathrm{~s})$ groups was statistically significant by a student's $t$ test though clinically it was not very significant.Mask ventilation was found to be easy in all patients in supine position whereas it was easy in only 30 patients in lateral position. In 6 patients, it was found to be difficult but adequate as defined by either requirement of two persons, insertion of oral airway or increase in fresh gas flow to ensure adequate ventilation. In no patient was it totally inadequate requiring change over to supine position.

\section{Discussion:}

BURP maneuver is widely accepted as an aid to improve visualization during laryngoscopy. Takahata $\mathrm{O}$ et al studied effects of BURP maneuver on direct laryngoscopy ten years ago. They compared the visualization of the larynx using the BURP maneuver with that of laryngoscopy with and without simple laryngeal pressure (Back). The BURP maneuver significantly improved the visualization compared with that of the Back maneuver. It was also notable that there were no Grade IV patients after BURP maneuver. They concluded that the BURP maneuver improved the visualization of the larynx more easily than simple back pressure on the larynx. They also suggested that the BURP maneuver should be included in the routine methods used by anaesthesiologist for improving visualization of larynx. ${ }^{1}$

Wilson et al reported that this maneuver reduces incidence of failure to view any portion of glottis from about $9.2 \%$ to $1.6 \%^{2}$. Knill coined the term BURP maneuver to the application of Backward, Upward, Rightward Pressure on the larynx to aid its view. ${ }^{3}$ Takahata $\mathrm{O}$ et al studies effects of BURP maneuver on direct laryngoscopy. Six hundred and thirty patients without obvious malformation of the head and neck participated 
in this study. They divided the degree of visualization into five grades and compared the visualization of the larynx using the BURP maneuver with that of laryngoscopy with or without simple laryngeal pressure ("Back"). They concluded that the BURP maneuver improved the visualization of the larynx more easily than simple back pressure on the larynx. They also suggested that the BURP maneuver should be included in the routine methods used by anaesthesiologist for improving visualization of larynx. ${ }^{1}$

In our study, we compared supine position with lateral position. Mask ventilation was found to be easy in all patients in supine position whereas it was easy in only 30 patients in lateral position. In 6 patients $(8.3 \%)$, it was found to be difficult but adequate as defined by either requirement of two persons, insertion of oral airway or increase in fresh gas flow. In no patient was it totally inadequate requiring changeover to supine position. Conan et al performed a randomized controlled trial investigating the effects of left lateral positioning on airway anatomy and subsequent airway management. Laryngoscopic airway examination was performed in anaesthetized patients, in the supine and left lateral position, and in the presence and absence of cricoids pressure. The left lateral position resulted in a deterioration of laryngoscopic view in $35 \%$ of patients and improvements in none. ${ }^{4}$ Considering the difficulties associated with the intubation in lateral position and other recent availabilities of equipment such as laryngeal mask airway, the difficult airway society and other recent failed intubation drills do not mention airway management in lateral position ${ }^{5,6,7,8}$.

\section{Conclusion:}

Following induction of general anaesthesia ease of mask ventilation, laryngoscopy was done both in supine and lateral positions using Macintosh laryngoscope and the following conclusion were drawn. Mask ventilation tends to be difficult in lateral position when compared to supine and Laryngoscopic view tends to improve with BURP maneuver in both supine and lateral position.

Table 1: Comparison of ease ventilation by face mask between supine and lateral positions(n)

\begin{tabular}{|l|l|l|l|}
\hline & Easy & Difficult but adequate & Inadequate \\
\hline $\begin{array}{l}\text { Supine } \\
(\mathrm{n}=36)\end{array}$ & 36 & 0 & 0 \\
\hline $\begin{array}{l}\text { Lateral } \\
(\mathrm{n}=36)\end{array}$ & 30 & 6 & 0 \\
\hline
\end{tabular}

$\mathrm{P}$ Value $=0.0249$ SIGNIFICANT (BY FISHER EXACT TEST) Mask ventilation is significantly easier in supine than in lateral position.

Table 2: Laryngoscopictime(sec) student's t test

\begin{tabular}{|l|l|l|l|l|l|}
\hline & GROUP & $\mathbf{N}$ & Mean & Std. Deviation & T \\
\hline & Supine & 36 & 6.2500 & 2.22165 & 8.30000 \\
\hline & Lateral & 36 & 13.3056 & 4.59079 & $\mathrm{P}<0.001 \mathrm{vhs}$ \\
\hline
\end{tabular}

Statistically significant (student's t test ), clinically not significant

Table 6: Supine position improvement with and without burp

\begin{tabular}{|l|l|l|l|}
\hline & $\begin{array}{l}\text { SUPINE WITHOUT } \\
\text { BURP }\end{array}$ & SUPINE BURP & TOTAL \\
\hline 1 & $14(38 \%)$ & $30(86 \%)$ & $44(61 \%)$ \\
\cline { 1 - 2 } & & $6(14) \%$ & $22(30.5)$ \\
\cline { 1 - 1 } $2 \mathrm{~A}$ & $16(44.44 \%)$ & & $6(8.3 \%)$ \\
\hline 2B & & & $100 \%$ \\
\hline & $6(18 \%)$ & $100 \%$ & 100 \\
\hline
\end{tabular}

$\mathrm{X}^{2}=16.364 ;$ P VALUE 0.003 CHI SQUARE TEST

In supine position there was a very significant improvement in laryngoscopicgrade with the application of BURP maneuver. 
Table 7: Lateral position improvement with and without burp

\begin{tabular}{|l|l|l|l|}
\hline & $\begin{array}{l}\text { LATERAL WITHOUT } \\
\text { BURP }\end{array}$ & $\begin{array}{l}\text { LATERAL WITH } \\
\text { BURP }\end{array}$ & TOTAL \\
\hline 1 & $19(41.3 \%)$ & $30(86 \%)$ & $49(59.7 \%)$ \\
\hline $2 \mathrm{~A}$ & & & \\
\hline & $11(29.3 \%)$ & $6(14 \%)$ & $17(20.73 \%)$ \\
\hline 2B & & & \\
\hline & $16(34.7 \%)$ & & $16(19.5 \%)$ \\
\hline & & & $100 \%$ \\
\hline
\end{tabular}

$\mathrm{X}^{2}=$ 9.939; P VALUE 0.007( CHI SQUARE TEST). In lateral position there was a very significant improvement in laryngoscopic grade with the application of BURP maneuver

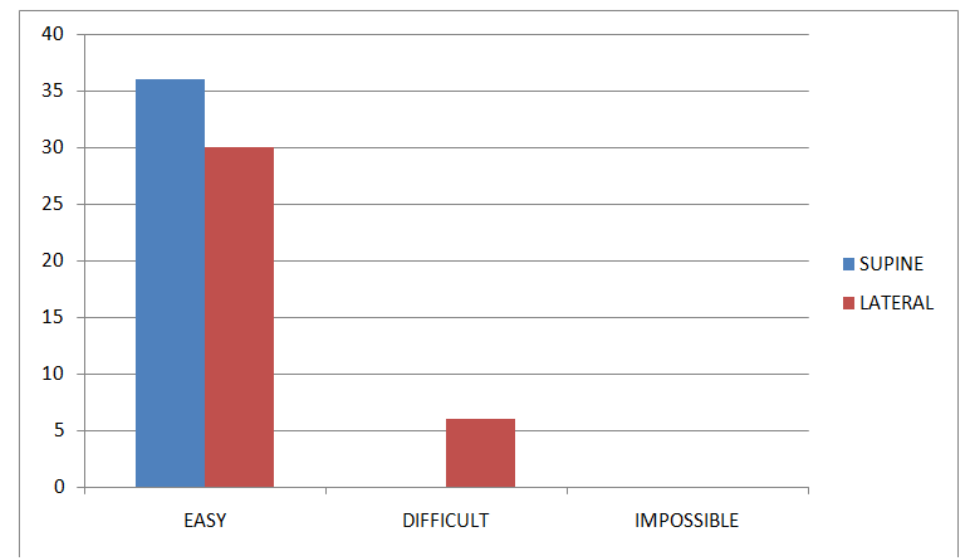

Figure 1: Comparison of ease of mask ventilation

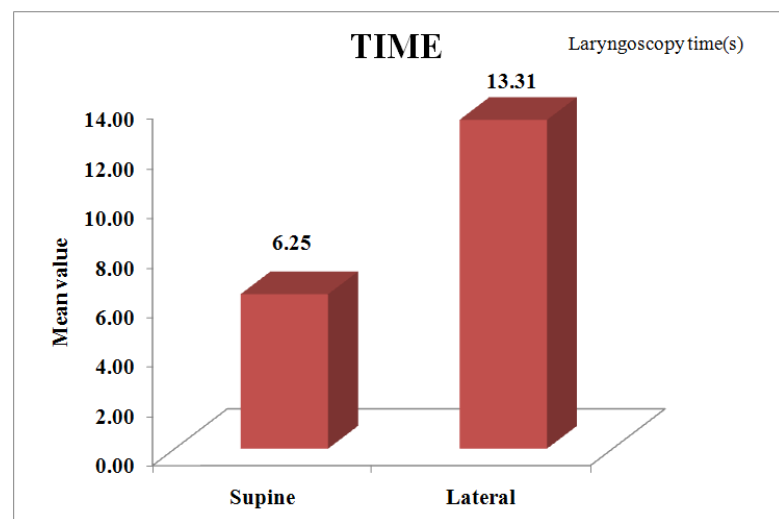

Figure 2: Comparison of laryngoscopy time: Time taken for laryngoscopy(s) in supine and lateral positions

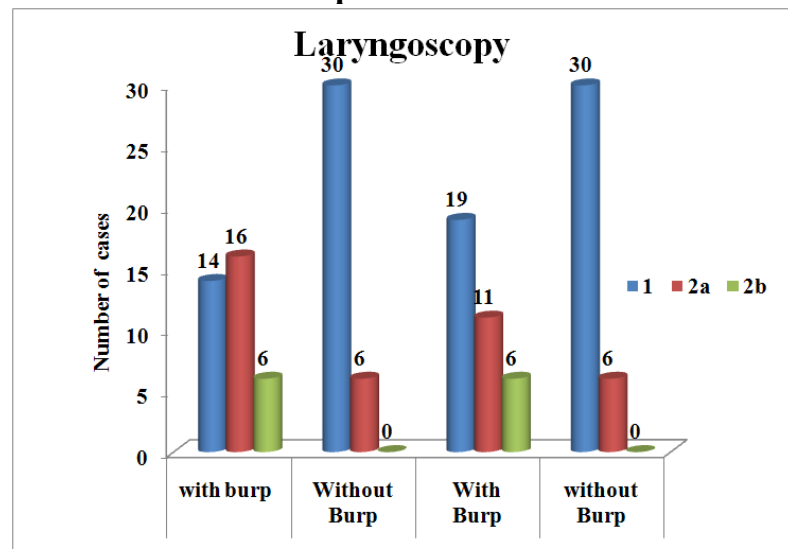

Figure 3: Comparison of laryngoscopic view with and without BURP in supine and lateral position 


\section{References:}

[1]. Takahata O, Kubota M, Mmiya K, et al. The efficacy of the "BURP" maneuver during a difficult laryngoscopy. AnesthAnalg 1997;84:419-21

[2]. Wilson M, Spiegelhalter D, Robertson J, Lesser P. Predicting difficult intubation. Br. J Anaesth 1998;61:211-6.

[3]. Knill RL. Difficult laryngoscopy made easy with a "BURP". Can J Anaesth 1993; 40:279-82.

[4]. Conan LM, Harney D, Ryan M, Moran C, Kavanagh BP, Boylan JF. Airway management in lateral position: a randomized controlled trial. AnesthAnalg 2005;101:1221-5

[5]. Harner M. Difficult and failed intubations in obstetrics. Int. J ObstetAnesth 1997;6:25-31

[6]. Henderson JJ, Popat MT, Latto I P, Pearce AC. Difficult Airway Society guidelines for management of unanticipated difficult intubation. Anaesthesia 2004;59:675-94.

[7]. Kaldewey S. failed intubation drill in obstetrics. Int J ObstetAnesth 2007 (IN PRESS)

[8]. Nair A, Alderson JD. Failed intubation drill in obstetrics. Int J ObstetAnesth 2007 (IN PRESS). 